\title{
Articulações entre contracepção, sexualidade e relações de gênero
}

\section{Interconnections between contraception, sexuality, and gender relations}

\author{
Cristiane da Silva Cabral \\ Universidade de São Paulo. Faculdade de Saúde Pública. \\ São Paulo, SP, Brasil. \\ E-mail: cabralcsळusp.br
}

\section{Resumo}

O artigo propõe uma abordagem socioantropológica para discutir questões ainda candentes em torno da contracepção, sobretudo no contexto brasileiro. A prática contraceptiva compreende uma série de decisões e lógicas complexas entranhadas em múltiplos domínios da vida, requerendo análises acerca de práticas e representações sobre contracepção, maternidade, conjugalidade, família, prazer e sexualidade, sem que se deixe de considerar aspectos materiais cruciais como a oferta contraceptiva (disponibilidade de serviços e métodos). São revisitadas algumas contribuições sociológicas e antropológicas que oferecem chaves de interpretação para o entendimento dos percursos contraceptivos femininos e permitem a discussão acerca dos processos decisórios relativos à contracepção. 0 artigo problematiza a equação simplista entre gestações imprevistas e falta de informação e/ ou acesso adequado aos métodos contraceptivos, ou ainda, como reflexo de uma suposta restrita racionalidade e/ou responsabilidade dos sujeitos em relação à vida contraceptiva - tendências ainda hegemônicas em campos disciplinares como a saúde pública e a demografia. As reflexões propostas ressaltam a necessidade de ponderar as assimetrias de gênero e as mediações biográficas, contextuais e culturais subjacentes nos processos que engendram um evento reprodutivo. Este tipo de análise abre espaço para introdução de discussões sobre sexualidade e sua conexão com as formas como as pessoas lidam com a contracepção e a reprodução. Trata-se de um artigo teórico que procura contribuir para o debate sobre a cultura contraceptiva no Brasil. Palavras-chave: Contracepção; Gênero; Sexualidade; Gravidez Imprevista; Métodos Contraceptivos; Reprodução.
Universidade de São Paulo. Faculdade de Saúde Pública. Av. Dr. Arnaldo, 715. São Paulo, SP, Brasil. CEP 01246-904. 


\section{Abstract}

This paper proposes a social-anthropological approach to discuss (still) burning issues around contraception, especially in the Brazilian context. Contraceptive practices embrace a wide range of decisions and complex perspectives intertwined in multiples life domains, requiring analysis about practices and representations on contraception, motherhood, conjugality, family, pleasure and sexuality, not forgetting the consideration of crucial material aspects as the contraceptive supply (availability of services and methods). Some sociological and anthropological contributions are revisited, since they provide interpretation keys to comprehend female contraceptives paths and enable the discussion about the decision-making processes associated to contraception. The article problematizes the simplistic equation which links unexpected pregnancy to the lack of information and/or proper access to contraception methods, or to an alleged and strict rationality and/or individual responsibility towards contraceptive life - this is a still hegemonic pattern in disciplinary fields such as Public Health and Demography. The presented considerations emphasize the urge to ponder biographical, contextual and cultural mediations and gender imbalance underlying a reproductive event. This kind of analysis enables to bring sexuality into discussion, and its relation with the ways people deal with contraception and reproduction. This is a theoretical article that aims to contribute to the debate about contraceptive culture in Brazil.

Keywords: Contraception; Gender; Sexuality; Unexpected Pregnancy; Contraceptive Methods; Reproduction.

\section{Introdução}

Muito se tem escrito sobre o tema da contracepção no Brasil, sobretudo nos campos disciplinares da saúde e da demografia. A abordagem geralmente incide sobre as conquistas em termos do crescente e progressivo acesso das mulheres aos métodos anticonceptivos, ou sobre seu contraponto, os ditos "fracassos" contraceptivos, tidos como expressão de dificuldades das mulheres quanto ao manejo da vida reprodutiva. Observa-se com frequência um tom que enfatiza a falta de recursos, em função de vários tipos de constrangimentos (sociais, simbólicos, culturais), associando episódios de gravidez imprevista ${ }^{1}$ ao manejo inadequado da contracepção por parte das mulheres. 0 argumento recorrente é de que tal inadequação teria raízes na precariedade do domínio da informação, perante as desvantajosas condições sociais e a baixa escolaridade das usuárias, bem como as dificuldades de acesso efetivo aos métodos anticonceptivos. Em outras palavras, é como se o manejo efetivo da contracepção - muitas vezes reduzido às ideias de informação e acesso aos métodos anticoncepcionais - permitisse total desvinculação entre sexualidade e reprodução. A resultante dessa equação seria a não ocorrência de gravidez imprevista na trajetória feminina. ${ }^{2}$

A literatura sobre contracepção também se caracteriza por um enfoque quase exclusivo sobre as mulheres, como se gravidez e contracepção não implicassem também os homens. Em parte, isso é efeito da não problematização das condições históricas específicas nas quais ocorre o intenso espraiamento dos anticonceptivos orais: se antes da pílula os métodos eram, sobretudo, masculinos (preservativo) ou relacionados ao ato sexual (coito interrompido, por exemplo), a partir do final da década de 1960 essa situação muda de forma drástica (Luker, 1975; Oudshoorn, 2003): a maioria dos métodos passa a ser dirigida às mulheres.

Uma consequência da mudança foi a diminuição da participação masculina nesse cenário. Por conseguinte,

\footnotetext{
1 Grande parte da literatura utiliza recorrentemente os termos gravidez "não planejada”, "indesejada”, "acidental”. Opto pelo termo gravidez imprevista (ou "não prevista”): trata-se de expressão cunhada em oposição às dimensões de planejamento e desejabilidade de uma gestação, presentes nas demais formas de classificação. Cf. Cabral (2011) para diferenciação e problematização acurada dos termos.

2 No Brasil, a proporção de nascimentos não planejados representava 50\%, em 1996, e 46\%, em 2006, segundo dados das Pesquisas sobre Demografia e Saúde correspondentes a tais períodos (Lago, 2015).
} 
assume-se que apenas as mulheres estão motivadas para a prática contraceptiva porque somente elas engravidam, obscurecendo-se que a gravidez é um fato social, não restrito à dimensão biológica. $\mathrm{O}$ apelo em relação à maior eficácia teórica dos métodos femininos (sejam os hormonais, o DIU ou a esterilização) rapidamente desliza para a concepção de que a responsabilidade pelo controle da fecundidade e de seus fracassos são femininos. Os homens se tornaram espectadores passivos das decisões contraceptivas, pelos mesmos processos (sociais e tecnológicos) que tornaram as mulheres agentes por excelência do processo reprodutivo (Darroch, 2008; Edwards, 1994; Luker, 1975; Oudshoorn, 2003).

Esse cenário cultural de maior responsabilização das mulheres pela contracepção e a correlata minimização da participação dos homens evidencia as assimetrias de gênero na esfera da reprodução. Entretanto, deve-se ter cautela para que o incremento da responsabilização feminina não seja interpretado pelo prisma da vitimização, com o argumento de que se amplia/intensifica a dimensão do trabalho reprodutivo das mulheres, tampouco pelo da celebração, quanto ao ganho de total autonomia feminina sobre o controle da fecundidade (Bajos; Ferrand, 2004; Baulieu; Heritier; Leridon, 1999; Haudiquet; Surduts; Tenenbaum, 2008).

Este artigo traz uma crítica à perspectiva majoritária da literatura que aborda a contracepção de um ponto de vista "dessexualizado", e ressalta a indissociabilidade das discussões sobre sexualidade e gênero para compreensão das práticas contraceptivas. Isso também significa mirar a contracepção menos como um dispositivo da biomedicina ocidental moderna, rapidamente associada à prevenção de riscos indesejáveis que podem advir do sexo (contraception as a medical good), e ressaltar sua centralidade para o exercício prazeroso da sexualidade (contraception as a sexual good) (Higgins; Smith, 2016; Sanders; Smith; Higgins, 2014; Sanders et al., 2001).

\section{Indissociabilidade entre sexualidade, contracepção e gênero}

Diversos estudos populacionais, realizados em diferentes países, indicam a predominância das práticas penetrativas nos encontros heterossexuais, em que o intercurso vaginal é considerado componente crucial (Bajos; Bozon, 2008; Heilborn; Cabral; Bozon, 2006; Laumann et al., 1994). Por conseguinte, as estratégias para evitar o engravidamento são constitutivas da heterossexualidade e a contracepção se faz necessária. Intimamente relacionadas, sexualidade e contracepção integram os roteiros sexuais aprendidos (Gagnon; Simon, 1973).

Alguns exemplos podem ser arrolados: a representação de que os homens possuem mais necessidades sexuais e conseguem controlar menos esses "impulsos" reforça a ideia de que a responsabilidade contraceptiva deve ser feminina (Heilborn; Cabral; Bozon, 2006; Salem, 2004); a noção de que as mulheres estariam mais propensas ao uso de métodos anticonceptivos em função de a gravidez ocorrer no corpo feminino; a crença de que as mulheres têm menos necessidades sexuais e, portanto, teriam maior controle de sua sexualidade; a representação corrente da espontaneidade das relações sexuais, em que se espera encontros íntimos intensos e espontâneos e, portanto, o uso de métodos como pílula anticoncepcional, DIU, ou esterilização, que separam o encontro sexual do ato da contracepção, seriam mais adequados a esse modelo hegemônico (Lowe, 2005; Spencer, 1999).

Mulheres e homens podem usar ou não contracepção. As razões dessa conduta extrapolam informação e acesso. É inegável que falta de conhecimento e/ou dificuldades de acesso aos métodos são fatores cruciais que impactam, em muito, a possibilidade de controle da fecundidade. Tais recursos, ou sua ausência, são com frequência elencados para caracterização dos contextos de decisões contraceptivas e reprodutivas. No entanto, é necessário salientar a existência de outros elementos nesse cenário, também intervenientes na (não) contracepção, que transcendem a tópica da informação e do acesso. Tal posicionamento significa tornar mais complexa essa equação (ainda) subjacente às políticas públicas, problematizar as discussões sobre gravidez imprevista, bem como inscrever a sexualidade nas discussões sobre contracepção (Higgins; Hirsch, 2007, 2008; Higgins; Smith, 2016).

O manejo contraceptivo é, definitivamente, um processo de múltiplos níveis, que interagem entre 
si. As práticas contraceptivas são influenciadas por: (1) fatores macroestruturais, tais como diferenças de classe social, normas de gênero e de sexualidade que caracterizam determinada cultura local, diferenças étnico-raciais, estruturas de serviços de saúde, políticas públicas etc.; (2) dimensões relacionais, que enquadram as lógicas de funcionamento e organização das parcerias afetivo-sexuais; e ainda, (3) elementos da trajetória individual (como contexto parental; preferências sexuais; intenções e/ou ambivalências em relação à reprodução etc.) (Bajos; Marquet, 200o; Higgins; Smith, 2016; Van Campenhoudt et al., 1997).

As práticas contraceptivas precisam ser compreendidas enquanto elemento indissociável das trajetórias afetivo-sexuais de homens e de mulheres. A abordagem aqui proposta sublinha outras concepções: (1) há uma socialização progressiva para a anticoncepção; (2) a experimentação amorosa e relacional é o cenário que enquadra o exercício e a socialização progressiva para a anticoncepção; (3) a problematização de certo racionalismo positivista que supõe um sujeito cognoscente, autônomo e reflexivo diante de cada atitude adotada (Caillé, 1998; Duarte et al., 1992) - aspecto subjacente a muitas abordagens sobre usos e fracassos contraceptivos. Portanto, é preciso levar às últimas consequências a ideia de que a prática contraceptiva, supostamente regular para as mulheres com vida sexual ativa, exprime as circunstâncias de relações de gênero, composição do par, moralidades sexuais, interações familiares, situação de classe, dimensões religiosas e aspectos geracionais, entre outras (Correa, Petchesky, 1996; Petchesky, 1984).

\section{Para além do desejo de evitar a gravidez: custos e benefícios da contracepção}

Apresento nesta seção os argumentos de algumas autoras que buscam romper com a análise calcada nos pressupostos racionalistas dos comportamentos contraceptivos. Trata-se de perspectivas teóricas socioantropológicas que ampliam a compreensão das práticas contraceptivas, para além de sua tradicional dimensão de (não) uso e acesso aos métodos.
Essas produções, realizadas em lugares e tempos distintos, ainda possuem grande rentabilidade interpretativa, sendo possível fazê-las convergir.

O advento tecnológico da pílula hormonal trouxe inúmeras transformações para a gestão da vida contraceptiva. Essa inovação permitiu às mulheres maior controle sobre a reprodução e foi interpretada, por um lado, como importante conquista delas no processo de busca pela igualdade entre homens e mulheres, pois as libertaria da maternidade compulsória. Por outro lado, algumas autoras contestam a ideia de que os procedimentos hormonais de controle da fecundidade tenham diminuído as diferenças de gênero (Bajos; Ferrand, 2004). É consensual, no entanto, que o advento da pílula anticoncepcional e a maior possibilidade de controle da reprodução passaram a ser parâmetro para os demais métodos. Desde então, a pílula e outros recursos hormonais, como os long acting reversible contraception, tendem a ser tidos como altamente eficazes, enquanto os demais métodos teriam maiores taxas de falha ou insucesso. Contudo, deve ser ressaltado o fato de que há diferentes critérios de escolha da contracepção, e que nem sempre a perspectiva do usuário coincide com a tônica dominante.

Há inúmeras formas de classificar os métodos contraceptivos. A lógica médico-sanitária inclinase a valorizar o critério de eficácia do método. Essa concepção tende a hierarquizar os diferentes métodos, segundo suas taxas de insucesso. Entretanto, a noção de eficácia de recursos contraceptivos pode ser relativizada, pois não só remete ao aspecto da probabilidade estatística do sucesso/fracasso (eficácia teórica mensurada em condições artificiais), como também à experiência pessoal dos indivíduos com cada método (eficácia prática). É possível optar pelo uso de um método contraceptivo estatisticamente pouco eficaz, que se revela muito satisfatório na trajetória contraceptiva pessoal. Exemplificando: os métodos tradicionais (como o coito interrompido) são geralmente retratados como possuidores de menor eficácia prática (pois dependem altamente da perícia do usuário). Entretanto, alguns estudos evidenciam sua expressiva eficácia quando utilizados por casais que declaram não desejar gravidez, e não passam 
por situações decorrentes de falha do método (Bajos et al., 2002). Outros autores argumentam que indivíduos ambivalentes quanto ao desejo de gravidez tenderiam a usar métodos associados ao ato sexual (também tidos como estatisticamente menos eficazes), pois favoreceriam a expressão dessa ambivalência (Bajos et al., 2002; Higgins; Hirsch; Trussell, 2008; Ortayli et al. 2005; Sanders et al., 2012; Trussell, 2011).

Bateman-Novaes (1979-1980, 1982a, 1982b, 2007) destaca que o critério de eficácia não é a única referência a presidir a opção por um método contraceptivo no plano individual. ${ }^{3}$ A escolha pode ser motivada pelo favorecimento ou não do prazer nas relações sexuais - seja pela conexão com a espontaneidade no ato sexual, quando é descartada a interrupção da prática sexual para o uso de um método, seja pela ideia de total disponibilidade para o sexo, em que a abstinência periódica não constitui uma opção. Assim, uma sexualidade sem entraves consistirá em critério relevante de escolha do método de contracepção. A autora afirma também que a avaliação em torno da pessoa que assume a responsabilidade da contracepção se configura como outro aspecto essencial na tomada de decisão sobre o tipo de método a ser utilizado. Ainda que seja corrente a representação de que a responsabilidade das questões contraceptivas e reprodutivas é feminina, há mulheres, homens ou casais, para os quais a autonomia da vida reprodutiva é um tema importante. De acordo com essa perspectiva, o uso do preservativo masculino ou do coito interrompido poderia sinalizar maior agenciamento masculino sobre o controle da reprodução, enquanto a pílula anticoncepcional ou o diafragma estariam relacionados ao protagonismo feminino nessa esfera. Segundo Bateman-Novaes (1979-1980, 1982a), nos casos em que a contracepção é assegurada por quem não detém a responsabilidade direta do uso do método, ele/a pode participar do cenário, ocupandose das providências necessárias. Citam-se como exemplo as situações em que o parceiro compra o contraceptivo para a mulher ou fornece o dinheiro para a aquisição, o que é frequentemente reportado por estudos qualitativos sobre o tema no Brasil (Cabral, 2003; Carvalho; Pirotta; Schor, 2000; Oliveira; Bilac; Muszkat, 2009).

A necessidade ou não de controle médico e acompanhamento especializado periódico também pode influenciar os critérios de escolha do método contraceptivo. Para algumas mulheres, o seguimento pode adquirir uma conotação negativa, sobretudo no caso de situações nas quais o acesso aos dispositivos institucionais de saúde é difícil - seja em função de constrangimentos econômicos, sociais ou por outras razões. Esse elemento está presente em parte dos discursos que revelam, no contexto brasileiro, forte rejeição ao DIU, aprendizado do uso da pílula anticoncepcional com amigas, obtenção do contraceptivo diretamente em balcão de farmácia ou, ainda, opção pela esterilização, que encerraria um ciclo de dependência da mulher em relação aos serviços de saúde (Cabral, 2011).

Bateman-Novaes (2007) indica que o binômio passividade-atividade é outro aspecto que circunscreve o uso de método contraceptivo, em que a possibilidade da iniciativa permite ao sujeito uma postura ativa (tomar pílula, usar camisinha, colocar diafragma) ou, ao contrário, não implica qualquer gesto particular. Além disso, os métodos contraceptivos podem ser classificados de acordo com as concepções de natural e artificial. Ainda que tais percepções sejam bastante variadas, a ideia central que motiva a escolha segundo esse tipo de critério é que não se deve impor ao corpo um método que altere seu funcionamento ou sua integridade. ${ }^{4}$

As considerações que Bateman-Novaes (19791980) tece a respeito da diversidade de atitudes e preferências pelos métodos vão no sentido de sublinhar que o uso da contracepção significa muito mais do que o desejo de evitar gestações. O comportamento contraceptivo se refere a escolhas

\footnotetext{
3 Simone Bateman-Novaes (1979-1980; 1982a; 1982b; 1985) entrevistou setenta de mulheres na França, em fins da década de 1970, que demandavam a interrupção da gestação.

4 Esse argumento é cada vez mais acionado no contexto francês, pelas gerações mais jovens, para justificar a rejeição ao uso da pílula anticoncepcional (Bajos et al., 2002, 2014). No Brasil, há um movimento crescente, observável sobretudo nas redes sociais, de mulheres que buscam métodos de contracepção não hormonais (green methods), com forte crítica à medicalização do corpo feminino e discussões sobre os malefícios que o uso de anticoncepcionais hormonais pode acarretar à saúde (Lima, 2017; Santos, 2017).
} 
de mulheres e homens, concernentes à vida sexual. Ao enfocar as trajetórias femininas, Bateman-Novaes (1979-1980) argumenta que "não querer ter um filho" não consiste em motivação suficiente para escolha e uso de determinado recurso contraceptivo. Nesse cenário incorrem outros fatores, como: o desejo de ela ter relações sexuais e com que frequência; a autoimagem da mulher (autônoma, ativa, passiva, disponível para o sexo etc.); o tipo de relacionamento em que está inserida (eventual; estável; mais ou menos igualitário); normas de gênero; moralidades sexuais; cultura prevalente na instituição médica, dentre outros.

Em certa medida, essa argumentação se aproxima das reflexões de Kristin Luker (1975) acerca dos custos e benefícios da contracepção e da reprodução. O trabalho dessa autora norte-americana pareceme até hoje proveitoso para a discussão dos nexos entre sexualidade e contracepção. Segundo Luker, o comportamento contraceptivo diz respeito não apenas ao controle da fecundidade, mas possui significados sociais e culturais, associados ao que a autora designa como "objetivos difusos" (diffused goals). Luker refuta a ideia de que a gravidez imprevista decorra da falta de habilidade contraceptiva ou da ausência de recursos para obter os métodos anticonceptivos. A autora recorre às teorias acerca do processo de tomada de decisão para argumentar em torno da existência tanto de racionalidade quanto de informação no contexto de uma gravidez imprevista: women are both informed and rational (Luker, 1975, p. 31). Para ela, a gravidez não prevista resulta de um processo decisório, em que há uma sequência de eventos e "riscos", situações nas quais as mulheres buscam atingir outros objetivos, mais difusos, do que simplesmente prevenir a gravidez. 5

0 argumento central da autora concerne à existência de custos e benefícios, tanto em torno da contracepção quanto da gravidez. Por um lado, a contracepção apresenta custos sociais, emocionais, financeiros e físicos, mas inteligíveis no contexto em que ocorrem. Por outro lado, as gestações imprevistas, inclusive aquelas com desfecho em aborto, possuem benefícios diversos e igualmente inteligíveis, dado o contexto no qual ocorrem. Tal perspectiva rompe com a representação hegemônica de que só há benefícios na gestão da contracepção e que uma gravidez imprevista é sempre indesejada ou negativa. A autora adverte que a avaliação de custos e benefícios da contracepção e da gravidez não significa considerar o sujeito da ação como exemplo cabal do cálculo racional. A dimensão subjetiva abarca intencionalidades, mas também as ambivalências (Luker, 1975).

$\mathrm{Na}$ contramão do pensamento hegemônico vigente à época, Luker (1975) se dedica a explicitar os custos associados à contracepção, dividindo-os em quatro grandes categorias: (1) os advindos dos significados sociais e culturais; (2) aqueles que derivam de problemas estruturais relacionados à contracepção; (3) os associados à manutenção da atividade contraceptiva (temporalidade); (4) os relacionados aos aspectos médicos e biológicos. Ela argumenta que, na contabilidade de custos e benefícios, as mulheres tendem a sopesar os custos atuais ou vigentes da contracepção, em relação às incertezas de uma gravidez que estaria no porvir. ${ }^{6}$

Dou alguns exemplos: as avaliações de gênero podem incidir de forma negativa sobre o comportamento contraceptivo, que, por sua íntima conexão com o sexo, carrega muitos significados da sexualidade, socialmente construída a partir de convenções culturais (leia-se também morais). Nesse sentido, quando realizada de forma contínua, a prática contraceptiva pode expressar a condição de pessoa sexualmente ativa, denotar planejamento do intercurso sexual, aludir à disponibilidade sexual feminina. A valorização do sexo como algo natural, fluido e espontâneo também pode imprimir dificuldades no manejo contraceptivo, seja para homens ou para mulheres. Não é incomum encontrar a representação (frequente em discursos nativos, segundo dados etnográficos) de que a relação

\footnotetext{
5 A análise empreendida pela autora situa-se no momento de efervescência do movimento feminista nos Estados Unidos. 0 estudo realizado em uma clínica de aborto legal na Califórnia enquadra-se em um projeto político de repensar as capacidades femininas de autonomia e reflexão. 0 tema, altamente controverso, do aborto como desfecho de um "comportamento irracional" das mulheres é o foco de sua análise. 6 Cf. Peretti-Watel (2010) para discussão sobre noção de "risco" enquanto categoria "redutora" das incertezas.
} 
sexual sem contracepção é "pura”, “natural”. Assim, as ideias de que a contracepção implicaria planejamento e o sexo pertenceria ao domínio da espontaneidade seriam concorrentes. A adoção de medidas de proteção prévias ao ato sexual poderia significar que a relação sexual está sendo esperada; em decorrência, o "calor" que derivaria da imprevisibilidade e espontaneidade seria reduzido, em função do cálculo racional inerente à contracepção.

Esses elementos remetem aos custos da contracepção e ilustram dificuldades que as mulheres podem enfrentar, sobretudo no início de suas trajetórias afetivo-sexuais. Reconhecerse sexualmente ativa pode implicar abrir mão da imagem de mulher ingênua e passiva, em prol de uma possível avaliação de que não se é mais virgem e, portanto, "inexperiente". A mulher pode "recusar" a contracepção para evitar transparecer a imagem do "tipo" disponível para o sexo. É recorrente nas narrativas das jovens, em estudos qualitativos, a enunciação dos obstáculos em adotar de forma contínua o uso da pílula anticoncepcional, por temerem que os pais, ao encontrarem a cartela de contraceptivo, descubram o exercício sexual regular. Manter a contracepção após o rompimento de um relacionamento afetivo-sexual também esbarra em estereótipos de gênero: pode significar que a mulher tem intenção de permanecer sexualmente ativa, a despeito do término da relação (Bajos et al., 2002; Beltzer; Bajos, 2008). Luker (1975) argumenta em termos de relationship-specific contraceptive pattern, ou seja, defende que as mulheres possuem padrões contraceptivos vinculados a cada tipo de relacionamento. Essa concepção é interessante para compreender o entrelaçamento de gênero, sexualidade e contracepção nas trajetórias afetivo-sexuais de mulheres, mas também de homens (Cabral, 2003, 2011; Oliveira; Bilac; Muszkat, 2009; Salem, 2004).

Em suma, a contracepção é raramente tematizada pelo prisma da díade ou das influências que a dimensão relacional e as preferências sexuais no âmbito da parceria afetivo-sexual podem exercer sobre o comportamento contraceptivo. McCave (2010) argumenta que essa falta de atenção e reflexão a respeito da dinâmica relacional explicaria porque algumas teorias são adequadas para predizer a intencionalidade de recorrer à contracepção, mas são inadequadas para predizer o uso real dela. Definitivamente, a sexualidade pode influenciar escolhas e práticas contraceptivas, e vice-versa.

\section{Momentos de vulnerabilidade: gravidez imprevista como objeto "bom para pensar"}

Outra interessante chave de leitura advém de um conjunto de reflexões sobre gestão da contracepção realizado por conceituadas autoras feministas francesas: Michèle Ferrand, Nathalie Bajos e colaboradoras. ${ }^{7}$ Essas autoras argumentam que o manejo ou o controle da fecundidade ocorre no entroncamento de diversas normas sociais, devendo ser analisados segundo distintos níveis, para que seja possível o entendimento da maneira como as mulheres gerenciam normas eventualmente contraditórias (Bajos; Ferrand; Hassoun, 2002, p. 35). Bajos et al. (2002) defendem que o contexto francês expressa a passagem de um modelo no qual a maternidade era "suportada" para um outro, em que a maternidade passa a ser "voluntária” e, teoricamente, controlada. Em outras palavras, o surgimento da contracepção moderna, sobretudo com o advento dos anticonceptivos orais, permitiu a montagem de um novo cenário no qual o nascimento de uma criança deve estar vinculado ao momento em que seus pais a desejam. Tal mudança foi possível pela instituição de uma "norma contraceptiva", caracterizada pela ampla difusão de métodos teoricamente eficazes de contracepção - a banalização do uso da pílula é um de seus corolários - e pelo estrito planejamento da prole.

\footnotetext{
7 Com o objetivo de investigar o aborto na trajetória feminina, um grupo de pesquisadores do Instituto Nacional de Saúde e Pesquisas Médicas e do Instituto Nacional de Estudos Demográficos desenvolveu um estudo em duas etapas na França. A primeira, qualitativa (pesquisa Gine), foi realizada em 1999 e 2000, a partir de entrevistas biográficas com oitenta mulheres que haviam tido uma gravidez imprevista nos últimos cinco anos. Em seguida, de 2002 a 2005, realizou-se um inquérito com aplicação de questionário a 7 mil mulheres, por telefone, sobre o itinerário contraceptivo e reprodutivo. A pesquisa se deteve em três grandes eixos de análise: acesso à contracepção e às falhas contraceptivas; processos de decisão diante de uma gravidez não prevista; eventuais desigualdades de acesso aos cuidados, nos casos de aborto.
} 
O processo político e sócio-histórico na França que enseja tais mudanças tem como atores centrais não só o movimento feminista, mas também o corpo médico na constituição, difusão e consolidação dessa "norma contraceptiva”. No contexto francês, as mulheres que não usam contraceptivos modernos e não desejam ficar grávidas são vistas como desviantes. Esse modelo ideal e hegemônico também prescreve métodos contraceptivos para cada fase da trajetória sexual: com a epidemia de HIV/Aids, incorporou-se o uso do preservativo no início dos relacionamentos afetivosexuais; passa-se a utilizar a pílula tão logo a relação se estabilize. O término da carreira reprodutiva é caracterizado pela utilização do DIU, assim que o número de filhos desejados é alcançado.

No cenário francês, o recurso ao aborto pode aparecer como estratégia feminina de retorno à "normalidade" e adequação à "norma procriativa" (Bajos; Ferrand, 2006/1; Bateman-Novaes, 19791980, 1982a, 1985), sobretudo se a gravidez ocorre no início ou no final do ciclo reprodutivo (Sihvo et al., 2003). Legalizado na França desde 1975, o aborto não gerou queda na taxa de fecundidade do país, tampouco foi banalizado. Ele não substituiu a contracepção e as mulheres continuam a considerálo como último recurso. As autoras afirmam que a maternidade permanece preponderantemente pensada e vivida no quadro da conjugalidade e que as mulheres não desejam ter filhos sozinhas (Bajos; Ferrand, 2006/1). Portanto, a contracepção mantém estreito vínculo com modelos de família e conjugalidade vigentes na França.

Importa salientar que essa literatura sublinha as dificuldades das mulheres de praticar uma contracepção cotidiana ao longo de toda a vida reprodutiva - mesmo em sociedades com uma norma contraceptiva prescrita, consolidada e internalizada. As autoras assinalam que nem todas as mulheres possuem uma situação de domínio ou plena gestão de suas vidas, e que o posicionamento em relação à norma contraceptiva depende da inserção social, da fase da trajetória de vida e do contexto relacional vivenciado (Bajos; Ferrand; Hassoun, 2002). Essa literatura critica veementemente as perspectivas que buscam delinear os perfis das mulheres em risco de engravidar sem planejamento, postulando que se trata, de fato, de buscar entender os momentos de vulnerabilidade aos quais as mulheres estão submetidas, nos quais uma gestação pode sobrevir (Bajos; Ferrand; Hassoun, 2002).

Retomo, neste ponto, uma das questões levantadas no início deste artigo: a ocorrência de gestações imprevistas em patamares considerados ininteligíveis e/ou inadmissíveis, tendo em vista o arsenal contraceptivo (ou o conjunto de métodos) existente na atualidade. A perspectiva trazida neste artigo é a de que os eventos reprodutivos devem ser compreendidos segundo determinando contexto social e relacional. Dessa forma, a gravidez imprevista pode ser uma consequência direta da dinâmica relacional, um dos muitos componentes com os quais mulheres e homens precisam lidar para a gestão da heterossexualidade e da contracepção. Claro que as abordagens relacionais não devem abandonar a referência individual, mas é preciso considerar as experiências relacionais concretas vividas pelas mulheres, os constrangimentos culturais e as determinações sociais a que estão submetidas, para compreensão das gestações não previstas.

\section{Contracepção: tensões entre o individual e o relacional}

As limitações enfrentadas pelas mulheres no Brasil, sobretudo as mais pobres e menos escolarizadas, para obter acesso aos métodos contraceptivos modernos/medicalizados persistem (Brasil, 2009; Lago et al., 2016). Apresentam-se dificuldades de diversas ordens para acesso a um atendimento específico. A escolha do método anticoncepcional é frequentemente mediada pela experiência de amigas ou parentes. As farmácias privadas permanecem como principal fonte de obtenção de métodos reversíveis, a despeito dos esforços governamentais nessa esfera (Brasil, 2009; Lago et al., 2016). Não são desprezíveis os efeitos colaterais associados a determinados métodos, como a pílula e o DIU, que acarretam custos biológicos e físicos nas trajetórias contraceptivas femininas. Esses aspectos constituem as justificativas comuns para interrupções, descontinuidades e mudanças de métodos (Moreau et al., 2009). Entretanto, é preciso ir além e atentar para o argumento fundamental: se por um lado o ato sexual pode ser dissociado de suas 
consequências reprodutivas, por outro, o recurso à contracepção e a gestão da prática contraceptiva permanecem ligados às representações dos indivíduos sobre sexualidade.

As reflexões sociológicas de Luker (1975) e a literatura francesa sobre o tema da contracepção (Bajos; Ferrand, 2006/1; Bajos et al., 2002; BatemanNovaes, 1979-1980; 1982a; 1985) oferecem chaves de interpretação para o entendimento de práticas e percursos contraceptivos. A partir desses enfoques torna-se possível problematizar a equação simplista que associa gestações imprevistas à falta de informação e/ou acesso adequado aos métodos contraceptivos, ou ainda, a uma suposta restrita racionalidade e/ou (ir)responsabilidade dos sujeitos em relação à vida contraceptiva.

O argumento de que a contracepção está entranhada em um conjunto de relações sociais complexifica a ideia de que as práticas contraceptivas sejam de foro individual e feminino: diferentemente, enfatiza-se a centralidade das dinâmicas relacionais e dos distintos significados dos contextos afetivosexuais na gestão da contracepção. Esse aspecto é frequentemente ignorado pelas políticas públicas, que focalizam ações na perspectiva do "risco" e da responsabilização individual (Brandão; Cabral, 2017). Dito de outro modo, a contracepção se inscreve nos comportamentos sexuais, e conceber o "comportamento sexual a partir de uma lógica racionalista e pragmática significa desconsiderar os múltiplos vetores que se impõem ao indivíduo em suas escolhas, os quais se articulam a lógicas distintas" (Knauth et al., 2006).

A contracepção está inapelavelmente imbricada com a atividade sexual. Tal afirmação pode parecer óbvia, mas não o é. A literatura corrente em saúde pública e demografia caracteriza-se por uma espécie de assepsia sexual: tudo se passa como se a contracepção não estivesse imersa em um contexto de interação de sujeitos, no qual múltiplas facetas de um relacionamento íntimo influenciam escolhas e decisões sobre como evitar (ou não) uma gravidez. No debate em torno do fracasso contraceptivo e da gravidez imprevista, uma parcela considerável da literatura em geral privilegia aspectos concernentes aos constrangimentos sociais, relacionados à pobreza ou ignorância, pressupondo certo irracionalismo que dificultaria o enquadramento coerente das condutas. Ignora-se ainda que o manejo contraceptivo é um processo de múltiplos níveis. A literatura sobre o tema raramente se refere a essa tensão e tende a repetir o discurso da condição precária do sujeito social ignorante ou despossuído de agência, capital educacional e cultural etc.

As abordagens aqui apresentadas rompem com a matriz interpretativa tradicional e enfatizam a importância de considerar as mediações biográficas e contextuais subjacentes aos processos que engendram um evento reprodutivo. Mais do que isso, essas análises introduzem a perspectiva do exercício da sexualidade e sua conexão com a gestão da vida contraceptiva. Ampliam e complexificam a ideia de uso coerente e consistente da contracepção, pois o cotidiano é bastante plural em relação às normatizações das políticas públicas.

É na esteira das considerações teóricas expostas que este artigo busca contribuir para a discussão colocada neste dossiê sobre os desafios para a assistência farmacêutica em sua interface com o tema da contracepção de emergência (CE) no Brasil. São diversas as inquietações sociais e representações em torno do acesso e da utilização da CE, sobretudo por mulheres jovens. Já consideramos em outro momento (Brandão et al., 2017) que o debate sobre a CE coloca em relevo questões relativas a moralidades sexuais e hierarquias de gênero, ao trazer para a cena dimensões da agência e autonomia feminina, bem como disputas políticas e institucionais em torno da capacidade reprodutiva e da sexualidade feminina. São diversos os atores no cenário, e pouco tem sido desenvolvido sobre a figura dos profissionais de farmácia e a posição que ocupam na ampla rede/ teia de significados e normas sociais que se refere à contracepção, sexualidade e reprodução.

\section{Referências}

BAJOS, N.; BOZON, M. Enquête sur la sexualité en France: pratiques, genre et santé. Paris: La Découverte, 2008.

BAJOS, N.; FERRAND, M. La contraception, lévier réel ou symbolique de la domination masculine? Sciences Sociales et Santé, Paris, v. 22, n. 3, p. 117140, 2004. 
BAJOS, N.; FERRAND, M. L'interruption volontaire de grossesse et la recomposition de la norme procréative. Sociétés Contemporaines, Paris, n. 61, p. 91-117, 2006/1.

BAJOS, N.; FERRAND, M.; HASSOUN, D. Au risque de l'échec: la contraception au quotidien. In: BAJOS, N.; FERRAND, M.; équipe GINÉ. (Org.). De la contraception à l'avortement: sociologie des grossesses non prévues. Paris: Inserm, 2002. p. 33-78.

BAJOS, N.; MARQUET, J. Research on HIV sexual risk: social relations-based approach in a crosscultural perspective. Social Science \& Medicine, Oxford, v. 5o, n. 11, p. 1533-1546, 2000.

BAJOS, N. et al. De la contraception à l'avortement: sociologie des grossesses non prévues. Paris: Inserm, 2002.

BAJOS, N. et al. La crise de la pilule en France: vers un nouveau modele contraceptif? Population \& Sociétés, Paris, n. 511, p. 1-4, maio 2014.

BATEMAN-NOVAES, S. La grossesse accidentelle et la demande d'avortement. L'Année Sociologique, Paris, v. 3o, p. 219-241, 1979-1980.

BATEMAN-NOVAES, S. Corps fécondable, corps réfractaire? Dialogue, Paris, n. 76, p. 90-95, $1982 a$. (Le sexe et ses lois).

BATEMAN-NOVAES, S. Les récidivistes. Revue Française de Sociologie, Paris, v. 23, n. 3, p. 473485, $1982 \mathrm{~b}$.

BATEMAN-NOVAES, S. La procréation impossible. Dialogue, Paris, n. 87, p. 86-97, 1985. (Bioéthique et désir d'enfant).

BATEMAN-NOVAES, S. Contraception. In: MARZANO, M. (Coord.). Dictionnaire du corps. Paris: Presses Universitaires de France, 2007. p. 215-218.

BAULIEU, E. E.; HERITIER, F.; LERIDON, H. (Coord.). Contraception: contrainte ou liberté? Paris: Odile Jacob, 1999.

BELTZER, N.; BAJOS, N. De la contraception à la prévention: les enjeux de la négociation aux différentes étapes des trajectoires affectives et sexuelles. In: BAJOS, N.; BOZON, M.
(Coord.). Enquête sur la sexualité en France: pratiques, genre et santé. Paris: La Découverte, 2008. p. 437-46o.

BRANDÃO, E. R.; CABRAL, C. S. Da gravidez imprevista à contracepção: aportes para um debate. Cadernos de Saúde Pública, Rio de Janeiro, v. 33, n. 2, 2017. Disponível em: <https://goo.gl/ DGomfr $>$. Acesso em: $1^{0}$ nov. 2017.

BRANDÃO, E. R. et al. Os perigos subsumidos na contracepção de emergência: moralidades e saberes em jogo. Horizontes Antropológicos, Porto Alegre, v. 23, n. 47, p. 131-161, 2017.

BRASIL. Ministério da Saúde. Pesquisa Nacional de Demografia e Saúde da Criança e da Mulher PNDS 2006: dimensões do processo reprodutivo e da saúde da criança. Brasília, DF: Ministério da Saúde: Cebrap, 2009.

CABRAL, C. S. Contracepção e gravidez na adolescência na perspectiva de jovens pais de uma comunidade favelada do Rio de Janeiro. Cadernos de Saúde Pública, Rio de Janeiro, v. 19, p. 283-292, 2003. Suplemento 2.

CABRAL, C. S. Práticas contraceptivas e gestão da heterossexualidade: agência individual, contextos relacionais e gênero. 2011. Tese (Doutorado em Saúde Coletiva) - Instituto de Medicina Social da Universidade do Estado do Rio de Janeiro, Rio de Janeiro, 2011.

CAILLÉ, A. Nem holismo nem individualismo metodológicos: Marcel Mauss e o paradigma da dádiva. Revista Brasileira de Ciências Sociais, São Paulo, v. 13, n. 38, p. 5-38, 1998.

CARVALHO, M. L. O.; PIROTTA, K. C. M.; SCHOR, N. Apoio: a forma predominante de participação masculina na regulação da fecundidade do casal. Saúde e Sociedade, São Paulo, v. 9, n. 1-2, p. 61-76, 2000.

CORREA, S.; PETCHESKY, R. Direitos sexuais e reprodutivos: uma perspectiva feminista. Physis: Revista de Saúde Coletiva, Rio de Janeiro, v. 6 n. 1-2, p. 147-177, 1996.

DARROCH, J. E. Male fertility control: where are the men? Contraception, Los Angeles, v. 78, n. 4 , p. 7-17, 2008. 
DUARTE, L. F. D. et. al. Vicissitudes e limites da conversão à cidadania nas classes populares brasileiras. Revista Brasileira de Ciências Sociais, São Paulo, n. 22, 1992.

EDWARDS, S. R. The role of men in contraceptive decision-making: current knowledge and future implications. Family Planning Perspectives, Nova York, v. 26, n. 2, p. 77-82, 1994.

GAGNON, J.; SIMON, W. Sexual conduct: the social sources of human sexuality. Chicago: Aldine, 1973.

HAUDIQUET, V.; SURDUTS, M.; TENENBAUM, N. (Coord.). Une conquête inachevée: le droit des femmes à disposer de leur corps. Paris: Syllepse, 2008.

HEILBORN, M. L.; CABRAL, C. S.; BOZON, M. Valores sobre sexualidade e elenco de práticas: tensões entre modernização diferencial e lógicas tradicionais. In: HEILBORN, M. L. et al. (Org.). O aprendizado da sexualidade: reprodução e trajetórias sociais de jovens brasileiros. Rio de Janeiro: Fiocruz, 2006. p. 207-266.

HIGGINS, J. A.; HIRSCH, J. S. The pleasure deficit: revisiting the "sexuality connection" in reproductive health. International Family Planning Perspectives, Nova York, v. 33, n. 3 , p. 133-139, 2007.

HIGGINS, J. A.; HIRSCH, J. S. Pleasure, power and inequality: incorporating sexuality into research on contraceptive use. American Journal of Public Health, Stanford, v. 98, n. 10, p. 1803-1813, 2008.

HIGGINS, J. A.; HIRSCH, J. S.; TRUSSEL, J. Pleasure, prophylaxis and procreation: a qualitative analysis of intermittent contraceptive use and unintended pregnancy. Perspectives on Sexual and Reproductive Health, Nova York, v. 40, n. 3, p. 130-137, 2008.

HIGGINS, J. A.; SMITH, N. K. The sexual acceptability of contraception: reviewing the literature and building a new concept. Journal of Sex Research, Whitehall, v. 53, n. 4-5, p. 1-40, 2016.

KNAUTH, D. R. et al. Sexualidade juvenil: aportes para as políticas públicas. In: HEILBORN, M. L. et al. (Org.). O aprendizado da sexualidade: reprodução e trajetórias sociais de jovens brasileiros. Rio de Janeiro: Fiocruz, 2006. p. 400-417.

LAGO, T. G. Nascimentos não planejados no Brasil eram 46\% em 2006: diminuíram? Revista Brasileira de Estudos de População, v. 32, n. 2, p. 387-394, maio/ago. 2015.

LAGO, T. G. et al. Ouvindo mulheres: contracepção no município de São Paulo. São Paulo: Instituto de Saúde: Secretaria de Estado de Saúde de São Paulo, 2016. Disponível em: <https://goo.gl/ Mq3tkk>. Acesso em: 31 out. 2017.

LAUMANN, E. O. et al. The social organization of sexuality: sexual practices in the United States. Chicago: University of Chicago, 1994.

LIMA, J. D. Pílula anticoncepcional: da revolução sexual à revisão de seu uso. Nexo, São Paulo, 15 set. 2017. Disponível em: <https://goo.gl/cGyTVb〉. Acesso em: 31 out. 2017.

LOWE, P. Contraception and heterosex: an intimate relationship. Sexualities, Londres, v. 8, n. 1, p. 75-92, 2005.

LUKER, K. Taking chances: abortion and the decision not to contracept. Berkeley: University of California, 1975 .

MCCAVE, E. L. It is not just about me: a relational understanding of young women's sexual negotiations. Smith College Studies in Social Work, Northampton, v. 8o, n. 1, p. 53-69, 2010.

MOREAU, C. et al. Frequency of discontinuation of contraceptive use: results from a French population-based cohort. Human Reproduction, Oxford, v. 24, n. 6, p. 1387-1392, 2009.

OLIVEIRA, M. C.; BILAC, E. D.; MUSZKAT, M. Homens e anticoncepção: um estudo sobre duas gerações das 'camadas médias' paulistas. In: MIRANDA-RIBEIRO, P.; SIMÃO, A. B. Demografia em debate: qualificando os números: estudos sobre saúde sexual e reprodutiva no Brasil. Belo Horizonte: ABEP, 2009. v. 2, p. 275-311. 
ORTAYLI, N. et al. Why withdrawal? Why not withdrawal? Men's perspectives. Reproductive Health Matters, London, v. 13, n. 25, p. 164-173, 2005. OUDSHOORN, N. The male pill: a biography of a technology in the making. Durham: Duke University Press, 2003.

PERETTI-WATEL, P. La societé du risque. Paris: La Découverte, 2010.

PETCHESKY, R. P. Abortion and woman's choice: the state, sexuality, and reproductive freedom. Boston: Northeastern University, 1984.

SALEM, T. Homem... já viu, né? Representações sobre sexualidade e gênero entre homens de classe popular. In: HEILBORN, M. L. (Org.). Família e sexualidade. Rio de Janeiro: Fundação Getúlio Vargas, 2004. p. 15-61.

SANDERS, J. N.; SMITH, N. K.; HIGGINS, J. A. The intimate link: a systematic review of highly effective reversible contraception and women's sexual experience. Clinical Obstetrics and Gynecology, Hagerstown, v. 57, n. 4, p. 777-789, 2014.

SANDERS, S. A. et al. A prospective study of the effects of oral contraceptives on sexuality and well-being and their relationship to discontinuation. Contraception, Los Altos, v. 64, n. 1, p. 51-58, 2001.

SANDERS, S. A. et al. Condom use errors and problems: a global view. Sexual Health, Collingwood, v. 9, n. 1, p. 81-95, 2012.

SANTOS, A. C. A. "Adeus, hormônios": concepções sobre corpo e contracepção na perspectiva de mulheres jovens. In: MUNDOS DE MULHERES \& FAZENDO GÊNERO 11, 13., 2017, Florianópolis. Anais... Florianópolis: UFSC, 2017.

SIHVO, S. et al. Women's life cycle and abortion decision in unintended pregnancies. Epidemiology Community Health, Londres, v. 57, n. 8, p. 601-605, 2003.

SPENCER, B. La femme sans sexualité et l'homme irresponsable. Actes de la Recherche en Sciences Sociales, Paris, v. 128, n. 1, p. 29-33, 1999.

TRUSSELL, J. Contraceptive failure in the United States. Contraception, Los Altos, v. 83, n. 5, p. 397-404, 2011.

VAN CAMPENHOUDT, L. et al. (Ed.). Sexual interaction and HIV risk: new conceptual perspectives in European research. Londres: Taylor \& Francis, 1997. 